\title{
Mudanças temporais na estrutura vegetacional de um fragmento de Caatinga, sul do Piauí
}

Temporal changes in the vegetation structure of a Caatinga fragment, southern Piauí

\author{
L. S. Silva ${ }^{1 *}$; T. R. Costa ${ }^{1}$ N N. V. Salomão ${ }^{1}$; A. R. Alves ${ }^{2}$; T. R. Santos ${ }^{3}$; E. L. M. \\ Machado ${ }^{4}$ \\ ${ }^{1}$ Programa de Pós-graduação em Ciência Florestal, Universidade Federal dos Vales do Jequitinhonha e Mucuri \\ (UFVJM), 39100-000, Diamantina, MG, Brasil. \\ ${ }^{2}$ Centro de Ciências Agrárias, Universidade Federal Rural do Semi-Árido, Mossoró, RN, Brasil. \\ ${ }^{3}$ Universidade Federal do Piauí, 64900-000, Bom Jesus, Piauí. \\ ${ }^{4}$ Departamento de Engenharia Florestal, Universidade Federal dos Vales do Jequitinhonha e Mucuri (UFVJM), \\ 39100-000, Diamantina, MG, Brasil. \\ *leovandessoares@bol.com.br
}

(Recebido em 04 de novembro de 2019; aceito em 18 de fevereiro de 2020)

\begin{abstract}
Estudos de dinâmica florestal permitem avaliar as variações da composição florística, mortalidade, recrutamento e o crescimento das espécies, sendo que estas informações são importantes para conservação dos escossistemas florestais. $\mathrm{O}$ objetivo desse estudo foi avaliar as mudanças na estrutura de espécies arbustivas-arbóreas em uma área de Caatinga, na região sul do Piauí. Para realização desse estudo foi avaliado a estrutura da vegetação em dois períodos, o primeiro inventário florestal foi em 2016 e o segundo em 2019, utilizando para isso, o processo de amostragem do tipo sistemática com 13 unidades amostrais de $20 \times 20$ metros. Dentro das unidades amostrais foram mensurados e identificados todos os indivíduos vivos com CAP $\geq 6 \mathrm{~cm}$. Foram analisadas todas as mudanças ocorridas na vegetação entre os dois inventários, como número de indivíduos, famílias, espécies, índice de diversidade e equabilidade, dinâmica (recrutamento e mortalidade), estrutura e classes diamétricas. Nos resultados, houve decréscimo no número de indivíduos e o recrutamento foi inferior a mortalidade. A área basal do último inventário foi inferior a área basal inicial $\left(3,567-3,294 \mathrm{~m}^{2} \cdot \mathrm{h}^{-1}\right)$, devido ao número de indivíduos mortos. As mudanças foram mais aceleradas na primeira classe diamétrica, seja pela mortalidade ou crescimento para classes posteriores. A composição de espécies e a diversidade florística permaneceram inalteradas, a densidade dos indivíduos diminuiu e os sobreviventes aumentaram a área basal.

Palavras-chave: inventário continuo, mudanças estrurais, fitossociologia.
\end{abstract}

Forest dynamics studies allow to evaluate the variations in the floristic composition, mortality, recruitment and growth, this information is important for the conservation of the forest ecosystems. The objective of this study was to evaluate changes in the structure of shrub-tree species in an area of Caatinga, in southern Piauí. To carry out this study the vegetation structure was evaluated in two periods, the first forest inventory was in 2016 and the second in 2019, using for this, the systematic sampling process with 13 sampling units of $20 \times$ 20 meters. Within the sample units, all living individuals with CAP $\geq 6 \mathrm{~cm}$ and their respective heights were measured and identified. All changes in vegetation between the two inventories were analyzed, such as number of individuals, families, species, diversity and equability index, dynamics (recruitment and mortality), structure and diameter classes. In the results, there was a decrease in the number of individuals and the recruitment was lower than mortality. The basal area of the last inventory was lower than the initial basal area $\left(3.567-3.294 \mathrm{~m}^{2} \cdot \mathrm{h}^{-1}\right)$ due to the number of individuals killed. Changes were most accelerated in the first diameter class, either by mortality or growth to later classes. The species composition and floristic diversity remained unchanged, the density of the individuals decreased and the survivors increased the basal area.

Keywords: continuous inventory, structural changes, phytosociology.

\section{INTRODUÇÃO}

A Caatinga compreende um domínio morfoclimático que cobre a maior parte da região semiárida do nordeste brasileiro, e se distribui por uma área de cerca de $800.000 \mathrm{~km}^{2}$ [1]. A vegetação da Caatinga geralmente possui adaptações as condições de déficit hídrico, através da perda de folhas nos períodos de estiagem (plantas caducifólias) [2]. Possui também, sistemas de armazenamento de água em raízes e caules modificados e mecanismos fisiológicos adaptados [56]. Sua vegetação 
apresenta heterogeneidade em termos de composição florística e estrutura, resultado da elevada variação nas condições ambientais, climáticas e pedológicas, porém, é um dos tipos vegetacionais brasileiros menos conhecidos e mais negligenciados quanto à conservação de sua biodiversidade $[3,57]$. Somado a este fato, a pressão antrópica sobre a vegetação da Caatinga vem aumentando ao longo dos anos, com uma taxa de desmatamento de $0,23 \%$ ao ano [4].

Âreas de Caatinga vem sendo exploradas de forma ilegal e insustentável de forma acelerada e desordenada devido o consumo de lenha nativa para fins domésticos e industriais [58]. Isso tem levado a vegetação a diversos estágios de sucessão secundária, resultado de intervenções, por exemplo a prática do corte raso $[5,6]$, para fonte de energia e inserção de atividades agrícolas $[7$, 8]. Em consequência, grandes areas da Caatinga são ameaçadas por altas taxas de danos antrópicos [9].

Ápos o uso e exploração, a maioria dessas áreas são abandonadas e se regeneram naturalmente [10]. Riegelhaupt e Pareyn (2010) [11], afirmam que 25\% de toda demanda energética do comércio, indústria e do uso doméstico na região nordeste são extraídas das áreas naturais da Caatinga, restando apenas fragmentos isolados.

Uma vez que a fragmentação florestal é um dos primeiros aspectos mais evidentes das transformações de origem antrópica [60]. Isso tem levado a redução de áreas florestais contínuas em manchas ou fragmentos sujeitos a diferentes níveis de isolamento [12,13] e alterações na composição, funcionalidade e estrutura das comunidades.

Dessa forma, a composição florística e a estrutura da vegetação passam por mudanças ao longo do tempo. E para acompanhar essas mudanças é necessário o monitoramento através de parcelas permanentes, onde é possível avaliar tais alterações nos parâmetros de crescimento, recrutamento, mortalidade, aumento ou redução da área basal [14]. Por meio desses parâmetros é possível identificar os fatores que afetam as mudanças nessas comunidades vegetais [15]. Essas informações são essenciais para as ações de conservação e restauração das florestas.

Levando em consideração o histórico de antropização em um fragmento florestal, este estudo foi realizado com o objetivo de avaliar as mudanças na estrutura de espécies arbustivo-arbóreas em uma área de Caatinga, na região sul do Piauí. Partiu-se da hipótese que o número de espécies e a densidade de indivíduos variam com o tempo, e que essa variação não implicaria em significativas mudanças florísticas.

\section{MATERIAL E MÉTODOS}

\subsection{Descrição e histórico de uso da área}

Esse estudo foi realizado em um fragmento de Caatinga ( $\left.9^{\circ} 11^{\prime} 54.62^{\prime \prime} \mathrm{S} ; 44^{\circ} 26^{\prime} 24.29{ }^{\prime \prime} \mathrm{O}\right)$ em uma propriedade particular (Fazenda Angelical), localizada na zona rural do município de Bom Jesus, região sul do Piaú (Figura 1), à uma altitude média de $289 \mathrm{~m}$. O solo da área foi classificado como Latossolo Amarelo Distrófico [16].

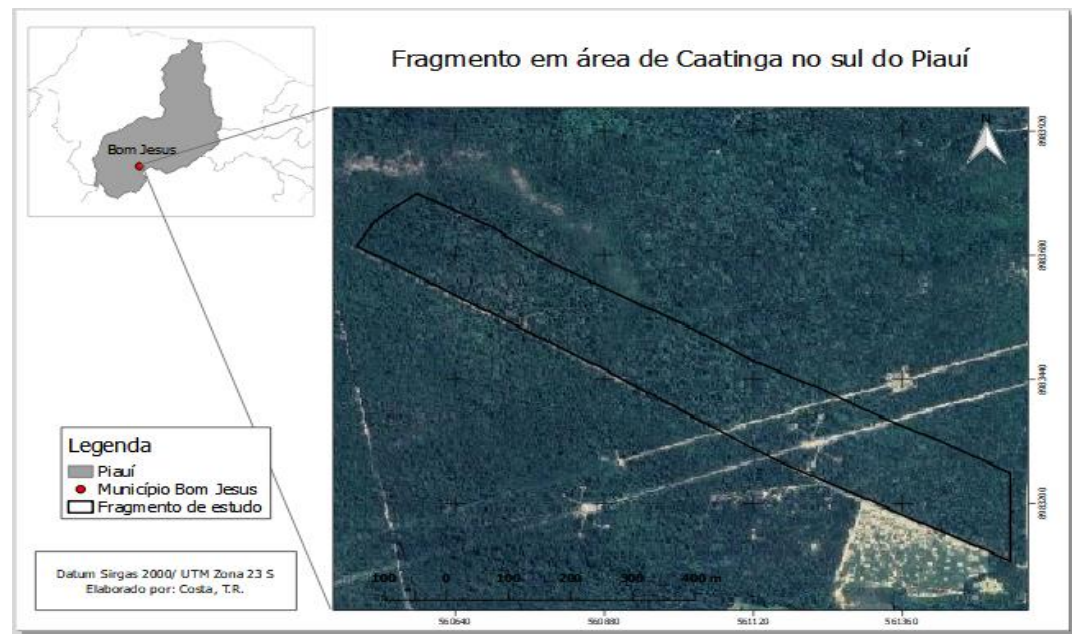

Figura 1. Mapa do Piauí e localização do município de Bom Jesus, onde se encontra a Fazenda Angical. 
Segundo a classificação de Köppen o clima da região é do tipo Aw (clima tropical com período seco de inverno), temperatura média $26,7^{\circ} \mathrm{C}$ e pluviosidade média anual de $1002 \mathrm{~mm}$ [17]. E apresenta dois períodos bem definidos, um período seco que compreende os meses de maio a outubro e um período chuvoso que ocorre entre novembro e abril [17].

A vegetação do fragmento foi submetida a um corte raso em toda a sua extensão no ano de 1993 , posteriormente foi abandonada e atualmente se encontra protegida sem intervenção antrópica e em processo de regeneração natural avançado. Porém há registro de trânsito de gado e duas linhas de transmissão que passam no interior do fragmento, além de ser circundada por pastagens.

\subsection{Monitoramento do compartimento arbustivo-arbóreo}

O primeiro inventário florestal no fragmento arbustivo-arbóreo foi realizado em 2016 e, para isso, foi utilizado o processo de amostragem sistemática. Nessa amostragem, somente a primeira unidade amostral é selecionada aleatoriamente, a partir dessa unidade amostral todas as demais são automaticamente selecionadas e distribuídas com espaçamento igual entre as unidades amostrais, segundo Soares et al. (2006) [59]. Assim, foram alocadas 13 parcelas permanentes de $20 \times 20 \mathrm{~m}$ $\left(400 \mathrm{~m}^{2}\right)$, distanciadas por $80 \mathrm{~m}$ entre si.

Na ocasião os indivíduos que atingiram o diâmetro estabelecido receberam placas numeradas, para serem monitorados, foram identificados e mensurados a circunferência de todos os indivíduos vivos com circunferência à altura do peito $(\mathrm{CAP}) \geq 6 \mathrm{~cm}$ [18]. Os indivíduos com troncos múltiplos foram mensurados e calculado o seu diâmetro equivalente.

As espécies quando não identificadas em campo foram coletadas exsicatas e posteriormente levadas para a identificação, sendo esta realizada por meio de consultas à literatura, por especialistas e se encontram depositadas no Herbário Dendrológico Jeanine Felfili (HDJF) da Universidade Federal dos Vales do Jequitinhonha e Mucuri (UFVJM). A classificação botânica seguiu o sistema APG IV [19].

Um segundo inventário foi realizado em 2019, neste foi utilizado os mesmos critérios usados no primeiro inventário. Os indivíduos sobreviventes foram mensurados novamente, os mortos registrados, e os novos indivíduos que atingiram o critério mínimo de inclusão (recrutas) (CAP) $\geq$ $6 \mathrm{~cm}$ foram identificados, receberam placas com numeração e tiveram mensurados os diâmetros.

\subsection{Dinâmica da comunidade arbustiva-arbórea}

Foram calculadas as taxas anuais médias de mortalidade $(\mathrm{M})$, recrutamento $(\mathrm{R})$ perda $(\mathrm{P})$ e ganho (G) de área basal das árvores por meio de expressões exponenciais [20, 21].

$$
\begin{aligned}
& \mathrm{M}=\left\{1-\left[\left(\mathrm{N}_{0}-\mathrm{m}\right) / \mathrm{N}_{0}\right]^{1 / \mathrm{t}} \times 100 ; \mathrm{R}=\left[1-\left(1-\mathrm{r} / \mathrm{N}_{\mathrm{f}}\right]^{1 / \mathrm{t}} \times 100 ;\right.\right. \\
& \mathrm{P}=\left\{1-\left[\left(\mathrm{AB}_{0}-\mathrm{AB}_{\mathrm{m}} \mathrm{AB}_{\mathrm{d}}\right) / \mathrm{AB}_{0}\right]^{1 / \mathrm{t}}\right\} \times 100 ; \\
& \mathrm{G}=\left\{1-\left[1-\left(\mathrm{AB}_{\mathrm{r}}+\mathrm{AB}_{\mathrm{g}}\right) / \mathrm{AB}_{\mathrm{f}}\right]^{1 / \mathrm{t}}\right\} \times 100
\end{aligned}
$$

Em que: $\mathrm{N}_{0}$ e $\mathrm{N}_{\mathrm{f}}=$ número de indivíduos inventário inicial e final respectivamente; $\mathrm{t}=$ tempo entre inventário inicial e final; $\mathrm{AB}_{0}$ e $\mathrm{AB}_{\mathrm{f}}=$ áreas basais dos indivíduos nos dois inventários; $\mathrm{AB}_{\mathrm{m}}$ e $A_{\mathrm{r}}=$ área basal dos mortos e ingressantes, respectivamente; $\mathrm{AB}_{\mathrm{d}}-\mathrm{AB}_{\mathrm{g}}=$ área basal do decremento e incremento.

Para expressar a dinâmica global, foram obtidas as taxas de rotatividade (turnover) em número de árvores (TN), a partir, das médias das taxas de mortalidade, recrutamento, perda e ganho, respectivamente $[22,23]$.

$$
\begin{aligned}
& \mathrm{TN}=(\mathrm{M}+\mathrm{R}) / 2 ; \\
& \mathrm{TAB}=(\mathrm{P}+\mathrm{G}) / 2
\end{aligned}
$$

Em que: $\mathrm{M}=$ taxa anual dos indivíduos mortos; $\mathrm{R}=$ taxa anual dos indivíduos recrutas; $\mathrm{P}$ e $\mathrm{G}=$ taxa de perda e ganho respectivamente.

Foram calculadas as taxas de mudança líquida no período, realizadas conforme Korning e Balslev (1994) [24] para área basal (ChAB) e para número de árvores.

$$
\begin{aligned}
& \mathrm{ChAB}=\left[\left(\mathrm{AB}_{\mathrm{f}} / \mathrm{AB}_{0}\right) 1 / \mathrm{t}-1\right] \times 100 \mathrm{e} \\
& \mathrm{ChN}=\left[\left(\mathrm{N}_{\mathrm{f}} / \mathrm{N}_{0}\right) 1 / \mathrm{t}-1\right] \times 100
\end{aligned}
$$


Em que: $\mathrm{t}=$ tempo entre o inventário inicial e final; $\mathrm{N}_{0}$ e $\mathrm{N}_{\mathrm{f}}=$ número de indivíduos inventário inicial e final, respectivamente; $\mathrm{AB}_{0}$ e $\mathrm{AB}_{\mathrm{f}}=$ áreas basais dos indivíduos primeiro e segundo inventário.

\subsection{Estrutura Horizontal}

Os parâmetros fitossociológicos da estrutura horizontal foram calculados conforme MuellerDombois e Ellemberg (1974) [25]: densidade relativa (DR), dominância relativa (DoR), frequência relativa (FR) e valor de importância (VI).

\subsection{Mudanças nas classes diamétricas}

Os indivíduos foram divididos em intervalos de classes diamétricas a partir do método de Sturges Finger (1992) [26]. Para interpretação das mudanças por classe de diâmetro foram contados os indivíduos que permaneceram na classe: mortos, recrutas, imigração (ingrowth) e emigração (outgrowth). A imigração e emigração podem ser progressivos ou regressivos [27]. Por meio de comparações entre contagens de Poisson foram calculadas as diferenças entre número de ingressos (recrutas + imigrantes) e indivíduos egressos (mortos + emigrantes) para cada classe de diâmetro.

A análise da riqueza florística foi realizada com auxílio do programa PAST 3.0 [28], sendo calculado os índices de diversidade de Shannon (H') e equabilidade de Pielou (J') [29].

\section{RESULTADOS E DISCUSSÃO}

No decorrer de três anos entre os dois períodos de avalição, observou-se que não houve mudanças na composição florística e no número de famílias (Tabela 1). Cavalcanti et al. (2009) [30]; Pimentel (2012) [31], em estudos de dinâmica na Caatinga, também observaram pouca ou nenhuma variação temporal na composição de famílias e de espécies. Portanto, esses resultados mostram que o período entre os inventários foi curto para detectar mudanças na composição florística. Isso pode estar relacionado ao nível de conservação do fragmento.

Os efeitos da perturbação de um determinado fragmento são demonstrados por meio de alterações na estrutura: altura, diâmetro, densidade e da dinâmica no habitat [32]. Quanto mais preservado o ambiente, diversas tendências estruturais são esperadas no desenvolvimento da sucessão ecológica, como o aumento da diversidade biológica, da equabilidade, uma vez que a comunidade adquire níveis estruturais mais complexos. Portanto, a ausência de distúrbio verificada ao longo dos anos para a área de estudo, possivelmente garante a manutenção da sua riqueza florística. Apesar de não ter ocorrido mudanças na composição florística e na diversidade taxonônica dessas plantas pode ser que tenha ocorrido mudanças nas características funcionais entre os períodos de amostragem.

$\mathrm{O}$ equilíbrio do número de espécies refletiu no índice de diversidade 2,60 nats.ind ${ }^{-1} \mathrm{e}$ equabilidade 0,75 , mantiveram os valores nos dois inventários (Tabela 1 ).

Tabela 1: Mudanças estruturais em um fragmento de Caatinga, na Fazenda Angical, Bom Jesus, PI.

\begin{tabular}{lcc}
\hline Parâmetros ecológicos & Inicial (2016) & Final (2019) \\
\hline Número de famílias & 11 & 11 \\
Número de espécies & 31 & 31 \\
Índice de diversidade de Shannon (H') & 2,60 & 2,60 \\
Equabilidade de Pielou (J) & 0,75 & 0,75 \\
\hline
\end{tabular}

Os valores de diversidade e equabilidade encontrados nesse estudo são considerados baixos, quando comparados com outros estudos em áreas de Caatinga no Sul do Piauí. Alves et al. (2013) [33] encontraram 2,96 nats.ind ${ }^{-1} \mathrm{em}$ um componente florestal no município de Bom Jesus. Os índices encontrados nesse estudo também diferiram dos resultados de Macedo et al. (2019) [34], que analisaram a composição e estrutura da vegetação preservada no sul do estado PI e encontraram 2,77 nats.ind ${ }^{-1}$. Similarmente, Vasconcelos et al. (2017) [35] encontraram 3,08 nats. ind ${ }^{-1}$ em um 
fragmento florestal no município de São Francisco-PI. Todos os resultados citados mostraram-se superiores ao encontrado nesse estudo, que possivelmente ainda seja reflexo do corte raso no qual esse fragmento foi submetido.

No entanto, quando comparado com áreas que sofreram o mesmo tipo de distúrbio antrópico, o valor do índice de diversidade nesse estudo foi superior ao encontrado por Calixto Junior e Drumond (2011) [36] 1,39 nats.ind ${ }^{-1}$, em um fragmento de Caatinga sensu stricto, analisado 30 anos após corte raso em Petrolina-PE, e por Sabino et al. (2016) [3] em duas áreas de Caatinga antropizadas na Paraíba encontraram 1,92 nats.ind ${ }^{-1}$, (área A) e 1,76 nats.ind ${ }^{-1}$ (área B). Isso porque o número de espécies encontrado nesse estudo foi superior ao número de especies desses estudos citados.

Conforme os dados apresentados na tabela 2, foram amostradas 11 famílias botânicas e 31 espécies em ambos inventários. A riqueza de espécies foi superior ao encontrado por Ferreira et al. (2016) [1], em uma área de Caatinga após 30 anos de supressão de vegetação em Pernambuco, e inferior ao encontrado por Macedo et al. (2019) [34] em uma área de Caatinga preservada no Sul do Piauí.

As famílias mais representativas foram Fabaceae 51,6\%, Combretaceae 6,4\% e Euphorbiaceae $6,4 \%$ das espécies amostradas em ambos inventários, respectivamente (Tabela 2).

Tabela 2: Lista de famílias/espécies lenhosas levantadas em fragmento de Faatinga, na Fazenda Angical, Bom Jesus, PI.

\begin{tabular}{ll}
\hline Família & Espécies \\
\hline Opiliaceae & Agonandra brasiliensis Miers ex Benth. \& Hook.f. \\
Apocynaceae & Aspidosperma pyrifolium Mart. \& Zucc. \\
Fabaceae & Bauhinia cheilantha (Bong.) Steud. \\
Moraceae & Brosimum gaudichaudii Trécul \\
Fabaceae & Cenostigma macrophyllum Tul. \\
Euphorbiaceae & Cnidoscolus quercifolius Pohl \\
Combretaceae & Combretum glaucocarpum Mart. \\
Combretaceae & Combretum leprosum Mart. \\
Fabaceae & Copaifera coriacea Mart. \\
Boraginaceae & Cordia rufescens A.DC. \\
Fabaceae & Dalbergia cearensis Ducke \\
Fabaceae & Diptychandra aurantiaca Tul. \\
Annonaceae & Ephedranthus pisocarpus R.E.Fr. \\
Erythroxylaceae & Erythroxylum laetevirens O.E.Schulz \\
Fabaceae & Hymenaea eriogyne Benth. \\
& Indeterminada 1 \\
& Indeterminada 2 \\
Malvaceae & Indeterminada 3 \\
Fabaceae & Luehea paniculata Mart. \& Zucc. \\
Fabaceae & Luetzelburgia auriculata (Allemão) Ducke \\
Euphorbiaceae & Machaerium sp. \\
Fabaceae & Manihot glaziovii Müll. Arg. \\
Fabaceae & Mimosa acutistipula (Mart.) Benth. \\
Fabaceae & Pityrocarpa moniliformis (Benth.) Luckow \& R.W.Jobson \\
Fabaceae & Peltogyne confertiflora Mart. ex Hayne) Benth. \\
Fabaceae & Pterodon abruptus (Moric.) Benth. \\
Fabaceae & Pterodon pubescens (Benth.) Benth \\
Fabaceae & Senegalia piauhiensis (Benth.) Seigler \& Ebinger \\
Fabaceae & Senegalia polyphylla (DC.) Britton \& Rose \\
Olacaceae & Swartzia flaemingii Raddi \\
\hline & Ximenia americana L. \\
\hline
\end{tabular}


Essas famílias também foram encontradas em outras áreas de Caatinga [3, 34-39]. Espécies da família Fabaceae possuem características morfológicas que fazem com que sejam adaptadas em locais sazonalmente secos. Segundo Freitas et al. (2011) [40] espécies da família Fabaceae em associação com bactérias do gênero Rhizobium exercem importante contribuição na fixação de nitrogênio no solo em um processo denominado fixação biológica de nitrogênio, disponibiliza nutrientes que contribuem para a melhoria da fertilidade dos solos na segião semiárida. Espécies da família Fabaceae são adaptadas às condições de baixa disponibilidade de água e altas temperaturas, portanto, elas são capazes de crescer e fixar nitrogênio com vantagem sobre espécies de outras familias [40].

Além disso, espécies dessa família geralmente apresentam dispersão anemocórica, que pode representar sucesso reprodutivo e colonização em ambientes abertos. Tais características conferem a essa família como uma das mais representativas em estudos de estrutura de comunidades vegetais [41, 42], enquanto espécies da família Combretaceae possuem imenso valor na medicina popular [43]. Do mesmo modo, Euphorbiaceae detém elevado valor econômico, devido ao múltiplo uso das espécies, que atendem a indústria farmacológica, extração de borracha, paisagismo, dentre outros [43]. Algumas espécies das famílias Combretaceae e Euphorbiaceae por apresentarem aspecto arbustivo ocorrem preferencialmente em ambientes abertos e secos como a Caatinga.

\subsection{Mudanças estruturais}

No intervalo entre os inventários ocorreram mudanças no número de indivíduos. Os indivíduos que morreram estão distribuídos em 11 espécies, porém 15 espécies não perderam indivíduos (Tabela 3).

As espécies que apresentaram os maiores valores de densidade, frequência, dominância relativa, e os maiores índices de valor de importância independentes no ano de amostragem foram: $C$. glaucocarpum, P. moniliformis, C. macrophyllum, C. leprosum e Machaerium sp. (Tabela 3). Isso confirma a predominância dessas espécies no fragmento de Caatinga. As espécies A. brasiliensis, $P$. pubescens, $P$. confertiflora e A. pyrifolium foram as que obtiveram os menores valores para esses parâmetros (Tabela 3).

Normalmente, as espécies estruturalmente de maior densidade e valor de importância são as mesmas ao longo do tempo em estudos na Caatinga [8, 31]. Quanto ao número de indivíduos, observou-se que existe grande concentração de indivíduos pertencentes a poucas espécies em relação às demais, como observados por Melo et al. (2019) [44]. Segundo Pereira et al. (2016) [45], a dominância de um grupo sobre outro é típica de floresta em estágio de regeneração.

As espécies $C$. glaucocarpum e $P$. moniliformis foram as que apresentaram maior número de indivíduos nos dois inventários, respectivamente (Tabela 3), que também aparecem entre as espécies de maior valor de importância em estudo realizado por Alves et al. (2013) [33] e Macedo et al. (2019) [34] em áreas de Caatinga. Foi observado o decréscimo do número de indivíduos nas populações de $C$. glaucocarpum e $P$. moniliformis, essa última foi a que mais perdeu indivíduos. Por outro lado, a espécie D. cearenses foi a que mais ganhou indivíduos pelo recrutamento (Tabela $3)$.

Segundo Lamprecht (1964) [46], o valor de importância é um parâmetro de integração parcial, que leva em consideração os valores relativos de dominância, densidade e frequência e mostra a importância ecológica relativa de cada espécie, melhor que qualquer outro parâmetro fitossociológico. Nesse contexto as cinco populações de maior valor de importância $(C$. glaucocarpum, P. moniliformis, C. macrophyllum, C. leprosum e Machaerium sp.) tiveram variação no número de indivíduos e no VI no segundo inventário $(67,64$ e 56,46\% do VI total, respectivamente), ou seja, uma maior contribuição na manutenção desse ambiente (Tabela 3).

Do ponto de vista ecológico, independentemente do tamanho populacional, todas as espécies apresentam importância para equilíbrio e manutenção do ambiente. Essa condição é ainda mais relevante em áreas fragmentadas e antropizadas, uma vez que a perda de uma determinada espécie pode levar a redução da diversidade e funcionalidade da comunidade. 
Tabela 3: Parâmetros fitossociológicos em um fragmento de Caatinga na Fazenda Angical, Bom Jesus, PI nos anos de 2016 e 2019. Os valores estão em ordem decrescente de número de indivíduo. Em que $N=$ número de indivíduos, $D R=$ Densidade Relativa (\%), $F R=$ Frequência Relativa (\%), AB = Área basal, DoR = Dominância Relativa $(\%), V I=$ Valor de Importância $(\%)$.

\begin{tabular}{|c|c|c|c|c|c|c|c|c|c|c|c|c|}
\hline Anos & & & & 016 & & & & & & 19 & & \\
\hline Espécies & NI1 & $\overline{\mathrm{DR}}$ & FR & $\mathrm{AB}$ & DoR & $\mathrm{VI} \%$ & NI2 & DR & FR & $\mathrm{AB}$ & DoR & $\mathrm{VI} \%$ \\
\hline C. glaucocarpum & 204 & 24,43 & 6,99 & 0,504 & 14,13 & 21 & 210 & 26,68 & 7,39 & 0,598 & 18,14 & 17,40 \\
\hline P. moniliformis & 110 & 13,17 & 6,45 & 1,1192 & 31,38 & 19,24 & 66 & 8,39 & 7,39 & 0,671 & 20,38 & 12,05 \\
\hline C. macrophyllum & 81 & 9,7 & 5,91 & 0,3426 & 9,61 & 9,67 & 82 & 10,42 & 6,82 & 0,401 & 12,17 & 9,80 \\
\hline C. leprosum & 98 & 11,74 & 5,91 & 0,3062 & 8,58 & 10,69 & 93 & 11,82 & 6,25 & 0,317 & 9,61 & 9,23 \\
\hline Machaerium sp. & 51 & 6,11 & 6,45 & 0,3181 & 8,92 & 7,04 & 54 & 6,86 & 6,82 & 0,338 & 10,27 & 7,98 \\
\hline P. abruptus & 26 & 3,11 & 5,91 & 0,1819 & 5,10 & 3,78 & 25 & 3,18 & 6,25 & 0,191 & 5,79 & 5,07 \\
\hline D. cearensis & 33 & 3,95 & 5,91 & 0,0635 & 1,78 & 3,23 & 44 & 5,59 & 5,68 & 0,090 & 2,74 & 4,67 \\
\hline S. piauhiensis & 38 & 4,55 & 5,38 & 0,0827 & 2,32 & 3,81 & 34 & 4,32 & 5,68 & 0,089 & 2,71 & 4,24 \\
\hline H. eriogyne & 29 & 3,47 & 3,23 & 0,0664 & 1,86 & 2,94 & 29 & 3,68 & 3,41 & 0,074 & 2,24 & 3,11 \\
\hline B. cheilantha. & 28 & 3,35 & 4,84 & 0,0357 & 1,00 & 2,57 & 21 & 2,67 & 4,55 & 0,027 & 0,82 & 2,68 \\
\hline C. coriacea & 14 & 1,68 & 4,84 & 0,0275 & 0,77 & 1,37 & 14 & 1,78 & 5,11 & 0,033 & 1,00 & 2,63 \\
\hline D. aurantiaca & 21 & 2,51 & 1,61 & 0,1064 & 2,98 & 2,67 & 20 & 2,54 & 1,70 & 0,111 & 3,36 & 2,53 \\
\hline Indet. 1 & 18 & 2,16 & 3,23 & 0,0355 & 1,00 & 1,77 & 17 & 2,16 & 3,98 & 0,044 & 1,33 & 2,49 \\
\hline P. pubescens & 1 & 0,12 & 5,91 & 0,0013 & 0,04 & 0,09 & 1 & 0,13 & 6,25 & 0,002 & 0,05 & 2,14 \\
\hline M. acutistipula & 9 & 1,08 & 3,23 & 0,0487 & 1,36 & 1,17 & 8 & 1,02 & 3,98 & 0,031 & 0,94 & 1,98 \\
\hline$X$. americana & 16 & 1,92 & 1,61 & 0,0495 & 1,39 & 1,74 & 15 & 1,91 & 1,14 & 0,051 & 1,54 & 1,53 \\
\hline S. flaemingii & 8 & 0,96 & 2,69 & 0,0192 & 0,54 & 0,82 & 9 & 1,14 & 2,27 & 0,026 & 0,78 & 1,40 \\
\hline E. pisocarpus & 10 & 1,2 & 5,91 & 0,0305 & 0,86 & 1,08 & 9 & 1,14 & 1,70 & 0,020 & 0,62 & 1,15 \\
\hline B. gaudichaudii & 3 & 0,36 & 1,61 & 0,1012 & 2,84 & 1,18 & 1 & 0,13 & 1,14 & 0,065 & 1,97 & 1,08 \\
\hline L. auriculata & 4 & 0,48 & 2,15 & 0,0112 & 0,32 & 0,42 & 4 & 0,51 & 2,27 & 0,015 & 0,44 & 1,07 \\
\hline Indet. 3 & 8 & 0,96 & 1,08 & 0,0208 & 0,58 & 0,83 & 6 & 0,76 & 1,70 & 0,019 & 0,57 & 1,01 \\
\hline L. paniculata & 4 & 0,48 & 1,61 & 0,0059 & 0,17 & 0,37 & 4 & 0,51 & 1,70 & 0,007 & 0,20 & 0,81 \\
\hline Indet. 2 & 5 & 0,6 & 1,08 & 0,0509 & 1,43 & 0,87 & 4 & 0,51 & 0,57 & 0,033 & 1,01 & 0,70 \\
\hline M. glaziovii & 3 & 0,36 & 1,08 & 0,0137 & 0,38 & 0,37 & 3 & 0,38 & 1,14 & 0,016 & 0,50 & 0,67 \\
\hline C. quercifolius & 3 & 0,36 & 1,61 & 0,0063 & 0,18 & 0,30 & 4 & 0,51 & 1,14 & 0,007 & 0,22 & 0,62 \\
\hline E. laetevirens & 2 & 0,24 & 0,54 & 0,0089 & 0,25 & 0,24 & 3 & 0,38 & 1,14 & 0,003 & 0,10 & 0,54 \\
\hline C. rufescens & 3 & 0,36 & 1,08 & 0,003 & 0,08 & 0,27 & 2 & 0,25 & 0,57 & 0,009 & 0,27 & 0,36 \\
\hline S. polyphylla & 2 & 0,24 & 0,54 & 0,0031 & 0,09 & 0,19 & 2 & 0,25 & 0,57 & 0,003 & 0,11 & 0,31 \\
\hline A. brasiliensis & 1 & 0,12 & 0,54 & 0,001 & 0,03 & 0,09 & 1 & 0,13 & 0,57 & 0,002 & 0,05 & 0,25 \\
\hline P. confertiflora & 1 & 0,12 & 0,54 & 0,001 & 0,03 & 0,09 & 1 & 0,13 & 0,57 & 0,001 & 0,04 & 0,25 \\
\hline A. pyrifolium & 1 & 0,12 & 0,54 & 0,0011 & 0,03 & 0,09 & 1 & 0,13 & 0,57 & 0,001 & 0,03 & 0,24 \\
\hline Total Geral & 835 & 100 & 100 & 3,567 & 100 & 100 & 787 & 100 & 100 & 3,294 & 100 & 100 \\
\hline
\end{tabular}

A espécie Combretum leprosum a quarta em valor de importância, geralmente é encontrada em estudos florísticos e fitossociológicos na Caatinga [33, 34, 47, 48]. A ocorrência dessa espécie em grande concentração de indivíduos é um indicativo que a vegetação se encontra em estágio de sucessão secundária [49].

No primeiro inventário em 2016 foram amostrados 835 indivíduos e no segundo em 2019 foram 787 indivíduos. Foi ainda registrado um decréscimo de 86 pela mortalidade e ganho de 38 indivíduos pelo recrutamento (Tabela 4).

A perda de indivíduos pela mortalidade foi superior ao ganho pelo recrutamento. Melo et al. (2019) [44], também observaram redução na densidade durante o monitoramento em área de Caatinga. Albuquerque et al. (2012) [5] e Ferreira et al. (2016) [1] atribuíram as baixas concentrações de chuvas e disponibilidade de água no solo como um dos fatores determinantes da redução populacional na Caatinga. 
Tabela 4: Mudanças estruturais em um fragmento de Caatinga na Fazenda Angical, Bom Jesus, PI.

\begin{tabular}{|c|c|}
\hline Número de árvores & \\
\hline Inicial (número de indivíduos) & 835 \\
\hline Final (número de indivíduos) & 787 \\
\hline Mortas (número de indivíduos) & 86 \\
\hline Recrutas (número de indivíduos) & 38 \\
\hline Sobreviventes (número de indivíduos) & 749 \\
\hline Taxa Mortalidade (\% ano- $\left.{ }^{-1}\right)$ & 5,3 \\
\hline Taxa Recrutamento (\% ano- $\left.{ }^{1}\right)$ & 2,4 \\
\hline Taxa Rotatividade (\% ano- $\left.{ }^{-1}\right)$ & 3,9 \\
\hline Taxa Mudança (\% ano-1) & $-2,9$ \\
\hline Área Basal & \\
\hline Inicial $\left(\mathrm{m}^{2} / \mathrm{ha}\right)$ & 3,567 \\
\hline Final $\left(\mathrm{m}^{2} / \mathrm{ha}\right)$ & 3,294 \\
\hline Mortas (m²/ha) & 0,482 \\
\hline Decremento dos sobreviventes $\left(\mathrm{m}^{2}\right)$ & $-0,258$ \\
\hline Recrutas (m²/ha) & 0,041 \\
\hline Crescimento Sobrevivência $\left(\mathrm{m}^{2} / \mathrm{ha}\right)$ & 0,468 \\
\hline Mudança líquida (\%) & $-3,34$ \\
\hline Taxa de perdas $\left(\%\right.$ ano- $\left.^{1}\right)$ & 11,12 \\
\hline Taxa de ganho (\% ano-1) & 8,05 \\
\hline
\end{tabular}

A taxa de mortalidade foi de 5,3\% ano- ${ }^{1}$, a exclusão dos indivíduos decorrente da mortalidade refletiu na área basal que reduziu de 3,56 para 3,29 m²/ha. Em uma área de Caatinga após 29 anos de supressão da vegetação [44], observaram aumento na área basal.

A taxa de perda $\left(11,12 \%\right.$ ano- $\left.^{-1}\right)$ foi superior a taxa de ganho $\left(8,05 \%\right.$ ano- $\left.{ }^{-1}\right)$, isso resultou em mudança líquida negativa $(-3,34)$. Mendivelso et al. (2013) [50], afirmaram que em florestas tropicais secas, as espécies vegetais possuem características funcionais (queda de folhas) em resposta à seca, o que resulta em diversidade de taxas de crescimento e indica que cada espécie pode ter estratégias diferentes para crescer sob diferentes condições de disponibilidade hídrica.

A diferença na área basal final não foi maior porque o incremento dos sobreviventes junto com os recrutas contribuiu com $0,468 \mathrm{~m}^{2} / \mathrm{ha}^{-1} \mathrm{e} 0,041 \mathrm{~m}^{2} / \mathrm{ha}^{-1}$, para a área basal final, respectivamente. Como foi uma área submetida ao corte raso, existem muitos indivíduos na mesma cepa, formando perfilhos, que com o tempo morrem provavelmente devido a competição ser maior entre os indivíduos na mesma cepa, o que pode ter contribuído para redução da área basal.

A distribuição dos indivíduos por classes de diâmetro em ambos os inventários teve tendência a $J$ reverso (Figura 2 e Tabela 5), com maior presença de indivíduos nas menores classes diamétricas que é considerada característica em florestas inequiâneas [51]. Isso demonstra que a comunidade se encontra com distribuição diamétrica balanceada, ou seja, a mortalidade é compensada pelo recrutamento dos indivíduos entre as classes diamétricas. Tal condição indica ainda uma comunidade estoque, com idade e composição de espécies variadas [52].

A primeira classe de diâmetro concentrou o maior número de indivíduos amostrados em ambos inventários. As mudanças foram mais acentuadas na primeira classe diamétrica, seja por mortalidade ou por mudanças para classes posteriores. Dos indivíduos que morreram 59,5\% estavam na primeira classe. Na primeira classe os egressos (emigrantes + mortos) foram superiores aos ingressantes (imigrantes + recrutas). Em todos os intervalos de classe a mortalidade ficou acima dos valores esperados, exceto a primeira classe. 


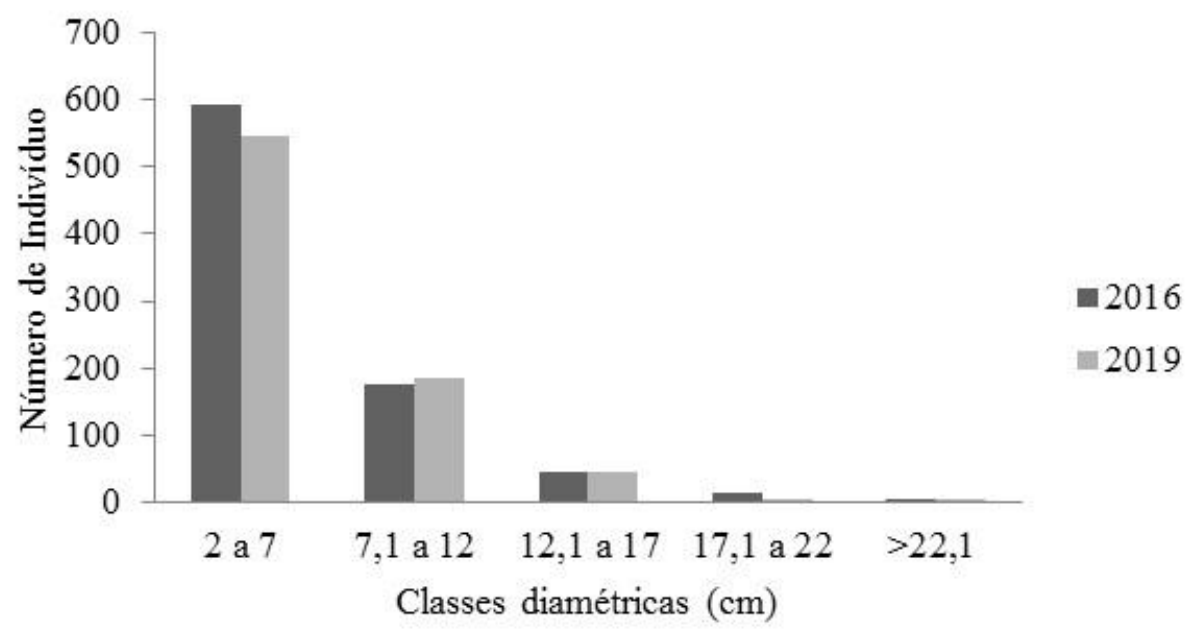

Figura 2. Distribuição diamétrica dos indivíduos amostrados em uma área de Caatinga na Fazenda Angical, Bom Jesus, PI, anos 2016 e 2019.

Tabela 5: Mudanças na estrutura diamétrica em uma área de Caatinga na Fazenda Angical, Bom Jesus, PI.

\begin{tabular}{llllllllllll}
\hline \multicolumn{1}{l}{$\mathrm{N}^{\mathrm{o}}$ de árvores } & \multicolumn{4}{c}{ Mortalidade } & Emig. & Rec. & Imig. & Cont. Poisson \\
\hline Classes & 2016 & 2019 & Esp. & $\mathrm{N}^{\circ}$ & Esp. & $\% \mathrm{ano}^{-1}$ & $\mathrm{~N}$ & $\mathrm{~N}^{\circ}$ & $\mathrm{N}^{\mathrm{o}}$ & $\mathrm{Z}$ & $\mathrm{p}$ \\
$2 \mathrm{a} 7$ & 594 & 545 & 559,85 & 51 & 61,18 & 3,22 & 40 & 38 & 8 & 3,84 & 0,001 \\
7,1 a 12 & 176 & 186 & 165,88 & 26 & 18,13 & 4,90 & 16 & 0 & 41 & 0,11 & $\mathrm{~ns}$ \\
12,1 a 17 & 45 & 46 & 42,41 & 7 & 4,63 & 5,35 & 10 & 0 & 18 & 0,17 & $\mathrm{~ns}$ \\
17,1 a 22 & 15 & 4 & 14,14 & 2 & 1,54 & 20,63 & 7 & 0 & 1 & 2,53 & 0,02 \\
$>22,1$ & 5 & 6 & 4,71 & 0 & 0,51 & 0,00 & 1 & 0 & 2 & 0,58 & $\mathrm{~ns}$ \\
Total & 835 & 787 & & 86 & & & 73 & 38 & 70 & & \\
\hline
\end{tabular}

Esp. = Frequências esperadas para o número de indivíduos no segundo inventário; $N=$ número de indivíduos mortos por classe de diâmetro, baseando-se na distribuição do número de indivíduos por classe de DAP, do primeiro inventário; Emig. = Taxas de mortalidade anual e os números de emigrantes; Rec = recrutas; Imig. = imigrantes, comparações de Poisson entre saídas (mortos + emigrantes) e entradas (recrutas + imigrantes).

A saída dos indivíduos por mortalidade ou para outras classes por meio do crescimento diamétrico, também foi maior na primeira classe. Já nas maiores classes as mudanças foram menos acentuadas. As mudanças foram significativas somente na primeira e quarta classe diamétrica.

Embora na primeira classe tenha ocorrido diminuição de indivíduos nesse intervalo de tempo, na segunda classe ocorreu o inverso, obteve aumento do número de indivíduos provenientes da primeira classe. Isso porque os indivíduos de diâmetros menores são mais susceptíveis a morrem devido a competição com indivíduos maiores [53], bem como crescimento para classes posteriores.

A competição interespecífica constituiu uma força reguladora importante nas taxas de dinâmica da comunidade estudada. Os resultados mostram que o fragmento se encontra em construção tardia. Segundo Guimarães et al. (2008) [54], isso ocorre devido os impactos ocorridos em anos anteriores, assim, ocorre mortalidade de indivíduos, incremento, recrutamento e aumento da área basal dos indivíduos sobreviventes (principalmente nas menores classes de diâmetro). Esse é um indicativo de que o fragmento está em processo de recuperação pós-distúrbio, sendo que o esperado para florestas em estágio avançado de sucessão é mortalidade independente da classe de diâmetro [55].

A ausência de mudanças na maioria dos parâmetros analisados (densidade, diversidade, riqueza, valor de importância) e as mudanças mínimas nas classes diamétricas intermediárias, permitem afirmar que a vegetação se manteve estável no tempo. Acredita-se que a intensidade dos impactos antrópicos não tenha sido suficiente para afetar negativamente os parâmetros avaliados, não configurando degradação e mantendo a comunidade no mesmo estágio sucessional. Bem como o intervalo entre os inventários foi curto dificultando o surgimento de respostas das populações de plantas estudadas. 


\section{CONCLUSÃO}

A hipótese das mudaças florísticas significativas foi rejeitada pelos resultados, ocorreram mudanças apenas na densidade dos indivíduos. Os padrões de dinâmica da comunidade mostram que a composição florística e diversidade de espécies arbustivo-arbóreas permaneceram inalteradas entre os inventários. A mortalidade foi superior ao recrutamento dos indivíduos. A distribuição diamétrica apresentou diminuição de indivíduos na primeira classe, as mudanças foram mais intensas nessa classe, tanto pela mortalidade quanto pelo crescimento dos indivíduos para classe posterior. A densidade dos indivíduos diminuiu e os sobreviventes aumentaram incremento em área basal.

\section{AGRADECIMENTOS}

Os autores agradecem o apoio da Coordenação de Aperfeiçoamento de Pessoal de Nível Superior (CAPES) no desenvolvimento deste trabalho, através de apoio financeiro e bolsas concedidas aos autores (L.S. Silva; T.R. Costa; N.V. Salomão). Ao Programa de Pós-Graduação em Ciência Florestal da Universidade Federal dos Vales do Jequitinhonha e Mucuri.

\section{REFERÊNCIAS BIBLIOGRÁFICAS}

1. Ferreira RLC, Silva SO, Silva JAA, Lira MA, Alves FTJR, Nascimento LM. Riqueza e diversidade de áreas da Caatinga em diferentes estágios sucessionais no nordeste do Brasil. Sci For. 2016;44(112):113. doi:10.18671/scifor.v44n112.02

2. Monteiro ER, Mangolin CA, Neves AF, Orasmo GR, Silva JGM, Machado MFPS. Genetic diversity and structure of populations in Pilosocereus gounellei (F.A.C.Weber ex K.Schum.) (Cactaceae) in the Caatinga biome as revealed by heterologous microsatellite primers. Biochem System Ecol. 2015;58(2):7-12, doi:10.1016/j.bse.2014.10.006

3. Sabino GS, Cunha MCL, Santana GM. Estrutura da Vegetação em Dois Fragmentos de Caatinga Antropizada na Paraíba. FLORAM. 2016;23(4):487-497, doi:10.1590/2179-8087.017315

4. IBAMA - Instituto Brasileiro de Meio Ambiente e Recursos Naturais Renováveis. Projeto de Monitoramento do Desmatamento dos Biomas Brasileiros por Satélite - PMDBBS: Caatinga. 2016. Disponível em: Acesso em: out. 2019.

5. Albuquerque UP, Araújo EL, El-Deir ACA, Lima ALA, Souto A, Bezerra BM, Ferraz EMN, Freire EMX, Sampaio EVSB, Las-Casas FMG, Moura, GJB, Pereira GA, Melo JG, Ramos MA, Rodal, MJN, Schiel N, Lyra-Neves RM, Alves RRN, Azevedo-Junior SM, Júnior WRT, Severi W. Caatinga Revisited: Ecology and Conservation of an Important Seasonal Dry Forest. Sci World J. 2012;1-18, doi:10.1100/2012/205182

6. Brunet J, Frenne P, Holmström E, Mayr ML. Life-history traits explain rapid colonization of young postagricultural forests by understory herbs. For Ecol Manag. 2012;278:55-62.

7. Drumond MA, Pires IE, Oliveira VR, Oliveira AR, Alvarez IA. Produção e distribuição de biomassa de espécies arbóreas no semiárido brasileiro. Rev Árvore. 2008;2(4):665-669, doi:10.1590/S010067622008000400007

8. Barreto TNA. Dinâmica de espécies lenhosas em área de Caatinga, Floresta PE. [Dissertação]. Mestrado em Ciências Florestais. Universidade Federal Rural de Pernambuco, Recife, 2013. 86 p.

9. Griscom HP, Ashton MS. Restoration of dry tropical forests in Central America: A review of pattern and process. For Ecol Manag. 2011;226(10):1564-1579, doi:10.1016/j.foreco.2010.08.027

10. Andrade JR, Silva KA, Santos JMF, Santos DM, Guerra TP, Araújo EL. Influence of microhabitats on the performance of herbaceous species in areas of mature and secondary forest in the semiarid region of Brazil. Rev Biol Trop. 2015;63(2):357-368.

11. Riegelhaupt EM, Pareyn FGC. A questão energética e o manejo florestal da Caatinga. In: Gariglio MA, Sampaio EVSA, Cestaro LA, Kageyama PY. (Org.). Uso Sustentável e conservação dos recursos florestais da Caatinga, Brasília: Serviço Florestal Brasiliero, 2010; 65-75.

12. Steffen W, Grinevald J, Crutzen P, McNeill J. The Anthropocene: conceptual and historical perspectives. Philos Trans R Soc A. 2011;369(1938):842-867.

13. Cruz AP, Higuchi P, Silva AN, Kilca RV, Dallabrida JP, Souza K, Lima LC, Soboleski VF, Nunes AS, Loebens R. Ci Fl. 2018;28(1):67-79, doi:10.5902/1980509831579

14. Figueiredo LTM, Soares CPB, Souza AL, Martins SV. Alterações florísticas em uma floresta estacional semidecidual no município de Viçosa, MG, entre 1994 e 2008. FLORESTA. 2013;43(2):169-180, doi:10.5380/rf.v43i2.28869 
15. Magalhães JHR, Júnior JAP, Vale VS, Schiavini I, Dinâmica do estrato arbóreo em uma floresta estacional semidecidual em Uberlândia, Minas Gerais, Brasil. Iheringia. 2017;729(3):394-402.

16. EMBRAPA - Sistema Brasileiro de Classificação de Solos. O Novo mapa de solos do Brasil: legenda atualizada / Humberto Gonçalves dos Santos ... [et al.]. — Dados eletrônicos. — Rio de Janeiro: Embrapa Solos, 2011. 67p.

17. Alvares CA, Stape JL, Sentelhas PC, Gonçalves JLM, Sparovek G. Köppen's climate classification map for Brazil. Meteorol Z. 2013;22(6):711-728.

18. Comitê Técnico Científico da Rede de Manejo FlorestaL da Caatinga. Rede de manejo florestal da Caatinga: protocolo de medições de parcelas permanentes. Recife: Associação Plantas do Nordeste. 2007. 28p.

19. APG IV - Angiosperm Phylogeny Group. An update of the Angiosperm Phylogeny Group classification for the orders and families of flowering plants: APG IV. Bot J Linn Soc. 2016;181:1-20.

20. Sheil D, Jennings S, Savill P. Long-term permanent plot observations of vegetation dynamics in Budongo, a Ugandan rain forest. J Trop Ecol. 2000;16(6):765-800.

21. Sheil D, Burslem DFRP, Alder D. 1995. The interpretation and mis interpretation of mortality rate measures. J Ecol. 1995;83(2):331-333.

22. Oliveira-Filho AT, Mello JM, Scolforo JRS. Effects of past disturbance and edges on tree community structure and dynamics within a fragment of tropical semideciduos forest in south-eastern Brazil over a five year period (1987-1992). Plant Ecol. 1997;131(1):45-66.

23. Werneck M, Franceschinelli EV. Dynamics of a dry forest fragment after the exclusion of human disturbance in southeastern Brazil. Plant Ecol. 2004;174(2):337-346.

24. Korning J, Balslev H. Growth and mortality of trees in Amazonian tropical rain forest in Ecuador. J Veg Sci. 1994;4(1):77-86.

25. Mueller-Dombois D, Ellenberg H. Aims and methods of vegetation ecology. New York: John Wiley \& Sons 1974 ; p.547.

26. Finger CAG. Fundamentos de Biometria Florestal. Santa Maria: UFSM/CEPEF/FATEC, 1992; p. 269.

27. Lieberman D, Lieberman, M, Peralta, R, Hartshorn, GS. Mortality patterns and stand turnover rates in a wet tropical forest in Costa Rica. J Ecol. 1985;73(5):915-924.

28. Hammer $\varnothing$, Harper DAT, Ryan PD. PAST - Palaeontological statistics. 2013.

29. Brower JE, Zar JH. Field and laboratory methods for general ecology. Dubuque: WMC Brow. 1984; 28 p.

30. Cavalcanti ADC, Rodal MJN, Sampaio EVSB, Costa KCC. Mudanças florísticas e estruturais, após cinco anos, em uma comunidade de Caatinga no estado de Pernambuco, Brasil. Acta Bot Brasil. 2009;23(4):1210- 1212.

31. Pimentel DJO. Dinâmica de vegetação lenhosa em área de Caatinga, Floresta, PE. [Dissertação] Mestrado em Ciências Florestais - Universidade Federal de Pernambuco: Recife, 2012. 62 p.

32. Martorell C, Peters E. The measurement of chrocnic disturbance and its effects on the threatened cactos Mammillaria pectinifera. Biol Conserv. 2005;124:199-207.

33. Alves AR, Ribeiro IB, Sousa JRL, Barros SS, Sousa PR. Análise da estrutura vegetacional em uma área de caatinga no município de Bom Jesus, Piauí. Rev Caatinga. 2013;26(4):99-106.

34. Macedo WS, Silva LS, Alves AR, Martins AR. Análise do componente arbóreo em uma área de ecótono Cerrado-Caatinga no sul do Piaú, Brasil. Sci Plena. 2019;15(1):1-11, doi: 10.14808/sci.plena.2019.010201

35. Vasconcelos ADM, Henriques IGN, Souza MP, Santos WS, Ramos GG. Caracterização florística e fitossociológica em área de Caatinga para fins de manejo florestal no município de São Francisco-PI. ACSA. 2017;13(4):329-337.

36. Calixto Júnior JT, Drumond MA. Estrutura fitossociológica de um fragmento de caatinga sensu stricto 30 anos após corte raso, Petrolina-PE, Brasil. Rev Caatinga. 2011;24(2):67-74.

37. Souza LSB, Moura MSB, Sediyama GC, Silva TGF. Balanço de energia e controle biofísico da evapotranspiração na Caatinga em condições de seca intensa. Pesq Agropec Bras. 2015;50(8):627-636, doi:10.1590/S0100-204X2015000800001

38. Costa GM, Cardoso D, Queiroz LP, Conceição AA. Variações locais na riqueza florística em duas ecorregiões de caatinga. Rodriguésia. 2015;66(3):685-709.

39. Souza LSB, Moura MSB, Sediyama GC, Silva TGF. Balanço de energia e controle biofísico da evapotranspiração na Caatinga em condições de seca intensa. Pesq Agropec Bras. 2015;50(8):627-636, doi:10.1590/S0100-204X2015000800001

40. Freitas ADS, Silva TO, Menezes RSC, Sampaio EVSB, Araújo ER, Fraga VS. Nodulação e fixação de nitrogênio por forrageiras da caatinga cultivadas em solos do semiárido paraibano. Rev Bras Zootec. 2011;40(9):1856-1861. 
41. Apgaua DMG, Santos RM, Pereira DGS, Menino GCO, Pires GG, Fontes MAL, TNG DYP. Betadiversity in seasonally dry tropical forests (SDTF) in the Caatinga Biogeographic Domain, Brazil, and its implications for conservation. Biodivers Conserv. 2014;23:217-232, doi:10.1007/s10531-013-05999

42. Fyhrquist P, Mwasumbi L, Haeggström CA, Vuorela H, Hiltunen R, Vuorela P. Ethnobotanical and antimicrobial investigation on some species of Terminalia and Combretum (Combretaceae) growing in Tanzânia. J Ethnopharmacol. 2002;79:169-177.

43. Trindade MJS, Lameira OA. Espécies úteis da família Euphorbiaceae no Brasil. Rev Cubana Pl Medicinales. 2014;19(4):1-19.

44. Melo CLSMS, Ferreira RLC, Silva JAA, Machuca MAH, Cespedes GHG. Dynamics of dry tropical forest after three decades of vegetation suppression. Floresta e Ambiente. 2019;26(3):e20171163, doi:10.1590/2179-8087.116317

45. Pereira JLR, Andrade EM, Palácio HAQ, Raymer PCL, Filho R, Carvalho J, Pereira FJS. Carbon stocks in a tropical dry forest in Brazil. Rev Ci Agron. 2016;47:32-40, doi:10.5935/1806-6690.20160004

46. Lamprecht H. Ensayo sobre la estructura floristica de la parte sur-oriental dei Bosque Universitário: El Caimital, Estado Barinas. Rev For Venez. 1964;7(10-11):77- 119.

47. Alves LS, Holanda AC, Wanderley JAC, Sousa JS, Almeida PG. Regeneração natural em uma área de caatinga situada no Município de Pombal-PB - Brasil. Rev Verde. 2010;5(2):152-168.

48. Bessa MAP, Medeiros JF. Levantamento florístico e fitossociológico em fragmentos de Caatinga no Município de Taboleiro Grande-RN. Geotemas. 2011;1(2):69-83.

49. Rodal MJN, Martins FR, Sampaio EVSB. Levantamento quantitativo das plantas lenhosas em trechos de vegetação de caatinga em Pernambuco. Rev Caatinga. 2008;21(3):192-205.

50. Mendivelso HA, Camarero JJ, Royo Obregón O, Gutiérrez E, Toledo M. Respostas diferenciais de crescimento ao balanço hídrico de espécies de árvores de folha caduca coexistentes estão ligadas à densidade da madeira em uma floresta seca tropical boliviana. PLoS One. 2013;8(10):73855, doi:10.1371/journal.pone.0073855

51. Guedes RS, Zanella FCV, Júnior JEVC, Santana GM, Silva JA. Caracterização florísticofitossociológica do componente lenhoso de um trecho de caatinga no semiárido paraibano. Rev Caatinga. 2012;25(2):99-108.

52. Scolforo JR, Mello JM, Oliveira AD. (eds.). Inventário Florestal de Minas Gerais: Floresta Estacional Semidecidual e Ombrófila - Florística, Estrutura, Diversidade, Similaridade, Distribuição Diamétrica e de Altura, Volumetria, Tendências de Crescimento e Áreas Aptas para Manejo Florestal. Universidade Federal de Lavras, Lavras-MG. 2008; 1029 p.

53. Schwinning S, Weiner J. Mechanisms determining the degree of size asymmetry in competition among plants. Ecologia. 1998;113:447-455.

54. Guimarães JCC, Berg E Van Den, Castro, GC, Machado ELM, Oliveira-Filho AT. Dinâmica do componente arbustivo-arbóreo de uma floresta de galeria aluvial no planalto de Poços de Caldas, MG, Brasil. Braz J Bot. 2008;31(4):621-632, doi:10.1590/S0100-84042008000400008

55. Swaine MD, Lieberman D, Putz FE. The dynamics of tree populations in tropical forest: A review. J Trop Ecol. 1987;3(4):359-366.

56. Giulietti AM, Conceição A, Queiroz LP. Diversidade e caracterização das fanerógamas do Semiárido brasileiro. Recife: Associação Plantas do Nordeste. 2006; 488 p.

57. Farias SGG, Rodal MJN, Melo AL, Silva MAM, Lima ALA. Physiognomy and vegetation structure in different environments of "caatinga" in "Serra Talhada" Pernambuco state, Brazil. Ci Fl. 2016;26(2):435-448.

58. Ministerio do Meio Ambiente (MMA). Biomas Brasileiros. Disponível em: <https://www.mma.gov.br/biomas/caatinga>. Acesso em 20 Jan. 2020.

59. Soares CPB, Paula Neto F, Souza AL. Dendrometria e inventário florestal. Viçosa, MG: Universidade Federal de Viçosa, 2006. 276 p.

60. Reis CS, Conceição GM. Aspectos Florísticos de um Fragmento de Vegetação, localizado no Município de Caxias, Maranhão, Brasil. Sci Plena. 2010;6(2):1-16. 